\title{
Influence of Interaction Energy in Fluid-Fluid Phase Transitions on Langmuir Monolayers
}

\author{
Weber da Silva Robazzi* and Bernhard Joachim Mokross ${ }^{\dagger}$ \\ * Departamento de Engenharia de Alimentos, Centro Educacional do Oeste, \\ Universidade do Estado de Santa Catarina, R. Aracaju S/N, Centro, CEP: 89870-000, Pinhalzinho-SC, Brazil \\ ${ }^{\dagger}$ Departamento de Física e Ciência dos Materiais, Instituto de Física de São Carlos, \\ Universidade de São Paulo, Caixa Postal 479, CEP: 13560-970, São Carlos-SP, Brazil
}

Received on 4 December, 2005

\begin{abstract}
A pure lattice model for Langmuir monolayers is presented where only nearest neighbor interactions are considered. The flexibility of hydrophobic tails laying on the surface is taken into account but the segments above the surface are taken as upstanding rigid rods without contribution to the entropy. The numerical calculations show that to obtain more than one phase transition the flexibility of these tails has to be taken into account.
\end{abstract}

Keywords: Langmuir monolayers; Phase transitions; Lattice model

\section{INTRODUCTION}

Langmuir monolayers have been the subject of research for many years. Although most studies involve the elucidation of some experimental features of these systems, the number of theoretical papers dealing with the topic is increasing. The aim of most of these models is to describe the structure and phase transitions in Langmuir monolayers and to elucidate the different mechanisms involved in phase transitions. To achieve this aim the strategy is to reproduce the surface pressure vs. mean molecular area isotherms and compare them with experimental results. In a strict sense, theoretical models can be subdivided in two different classes: computer simulation models and molecular models.

The first class includes models that attempt to reproduce phase transitions in Langmuir monolayers in different ways: by including all atoms of the molecule forming the monolayer $[1,2,3]$ and by including only some selected degrees of freedom of these molecules [4,5].

The second class contains molecular models that attempt to reproduce phase transitions (inclusive tilting phase transitions) treating the molecules as cylindrical rods grafted on a two-dimensional lattice. An important restriction of these models is the use of lattices which imply the assumption that the mass center of the molecules are fixed on a hexagonal lattice. These models aim to explain the mechanism of successive fluid-fluid phase transitions and it is this problem that lays the main focus of interest of this paper.

Different assumptions have been made to explain the occurrence of more than one fluid-fluid phase transition. Some of them are based in a mean-field analysis using a lattice. Wang and Rice [6] employed a model similar to that of Scheutjens and Fleer [7]. They derived a system of nonlinear equations for the chain density profile normal to the surface and solving this system obtained the equation of state of the monolayer. In the model developed by Popielawski-Rice [8], different conformations of the molecules are treated as different species of molecules whose concentrations can be determined from the conditions of chemical equilibrium. They only obtained one fluid-fluid phase transition and it seems that the main reason that no more transitions showed up was the neglect of inter- actions between portions of the chains that are located at the space above the surface. Shin et al. [9] included those interactions in this model and obtained two transitions, which they interpreted as being due to a condensation of the portions of the amphiphilic molecules in the surface and due to a condensation of a "gas of tails" to a "liquid of tails" respectively. Cantor and McIlroy [10] improved the method developed by Flory [11] to determine the number of distinguishably different arrangements of the chains on the surface by accounting for the effects of orientational anisotropy. Even with this improvement, considering other models, they were not able to obtain two fluid-fluid phase transitions. Recently, Rusanov [12] proposed an equation of state based on the excluded area and obtained good agreement with experiments in a limited temperature range. Good agreement with experimental data were also obtained with the generalized Volmer equation $[13,14]$. By taking into account chain conformation and dipole-dipole interactions Ruckenstein and $\mathrm{Li}$ [15] were able to reproduce some characteristic features of isotherms of phospholipids.

In this work, we develop a lattice model in order to reproduce the fluid-fluid phase transitions in Langmuir monolayers adapting Flory's methodology to Langmuir monolayers. This implies in the inclusion of chain connectivities among the segments of the amphiphilic molecules that remain on the surface. The segments of the hydrophobic tail that remain above the surface are taken as rigid rods as shown in Fig. 1. Calculations show that flexibility of the tails is one of the main mechanisms responsible for fluid-fluid phase transitions as will be shown.

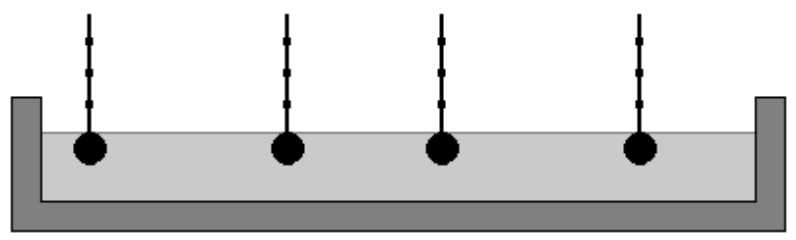

FIG. 1: Rigidity of chain segments on the air. 


\section{THE MODEL}

The model considers the whole system composed of surface, water and air as a cubic lattice. By hypothesis, each head group, water molecule, segment of the hydrophobic tail or air molecule may occupy only one lattice site. The sites belonging to the surface may be occupied by head groups, chain segments or water molecules. The polar head groups necessarily lay on one surface site. Each site of the layer located below the surface is occupied by one water molecule, and in the space above the surface are located the tail segments and/or the air molecules. For simplicity, we consider only nearest neighbors interactions.

\section{A. Analysis of the surface}

The surface is considered as a bidimensional lattice occupied by water molecules, hydrophobic segments and all polar head groups. The total number of surface lattice sites is $N_{0}$ and the total number of amphiphilic molecules is denoted by $M$. The molecules are assumed have $v$ segments (including the head group) and $N_{i}$ is the number of molecules that contain $i$ segments on the surface while the remaining $v-i$ are lifted on the space above the surface. The number of water molecules is denoted by $N_{w}$. The hydrophilic heads and the hydrophobic tail segments laying on the surface are considered alike at the counting of the different arrangements on the surface. The isothermal compression is done by stages: at each stage the number of sites is diminished by an amount of $\Delta N_{0}$. After each stage of compression the number of molecules remains the same, i.e.,

$$
\sum_{i=1}^{v} N_{i}=M
$$

And all sites of the surface are filled up,

$$
\sum_{i=1}^{v} i N_{i}+N_{w}=N_{0}
$$

The total number of arrangements of the molecules on the surface is given by the expression developed by Flory:

$$
\Omega_{T}=\frac{N_{0} !}{N_{w} ! \prod_{i=1}^{v} N_{i} !}\left[\frac{z-1}{N_{0}}\right]^{\sum_{i=2}^{v}(i-1) N_{i}}
$$

where $z$ is the coordination number of the lattice.

The total entropy, differently of many authors $[6,7,8,9,10]$ can not be done by applying Stirling's approximation, since during compression, some molecular species, given by $N_{i}$ 's, may assume small values invalidating this procedure that makes the approximation very poor. The proper way to calculate the entropy, is the evaluation of Boltzmann's expression without any simplification, i.e., the evaluation of

$$
\Delta S_{\text {Total }}=k_{B} \ln \Omega_{T}
$$

The interaction energy on the surface is obtained by a meanfield analysis which includes interactions only between nearest neighbors. The total interaction energy on the surface is given by:

$$
\Delta U_{S}=\Delta U_{w}+\sum_{i=1}^{v} \Delta U_{i}
$$

where $\Delta U_{w}$ and $\Delta U_{i}$ are given by:

$$
\begin{aligned}
\Delta U_{w} & =\frac{N_{w} z}{2 N_{0}}\left(\varepsilon_{h h} N_{w}+\varepsilon_{p h} \sum_{i=1}^{v} i N_{i}\right) \\
\Delta U_{i} & =\frac{i N_{i}}{2 N_{0}}\left(\varepsilon_{p h} N_{w}+\varepsilon_{p p} \sum_{i=1}^{v} i N_{i}\right)
\end{aligned}
$$

The parameters $\varepsilon_{h h}, \varepsilon_{p h}$ and $\varepsilon_{p p}$ denote the interaction energy between water molecules, water molecules and the amphiphile molecules and amphiphile molecules, respectively.

\section{B. Analysis of the layer below the surface}

The layer below the surface contains only water molecules and they interact with the molecules laying on the surface yielding

$$
\Delta U_{\text {down }}=N_{w} \varepsilon_{h h}+\varepsilon_{p h} \sum_{i=1}^{v} i N_{i}
$$

\section{Analysis of the layer above the surface}

As shown in Fig. 1, above the surface the hydrophobic tails are assumed as upstanding rigid rods. The interactions between these segments and the air molecules are not taken in account. Only rod-rod interactions are considered and

$$
U_{A i r}=U_{A i r 1}+U_{A i r 2}+\ldots+U_{A i r(v-1)}
$$

where

$$
U_{\text {Airj }}=\varepsilon_{p p}\left(M-\sum_{k=0}^{j-1} N_{v-k}\right)\left[1+\frac{z}{2 N_{0}}\left(M-\sum_{k=0}^{j-1} N_{v-k}\right)\right]
$$

The variables $U_{\text {Air } j}, \mathrm{j}=1,2,3, \ldots$ denote the energy of a segment in the $\mathrm{j}^{\text {th }}$ layer above the surface due to its interaction, $\varepsilon_{p p}$, with the segment in the layer just below it (belongs to the same molecule), and due to its interaction with the segments 
in the same layer belonging to other molecules. This last interaction is taken into account via a mean field approximation similar to what was done for the segments laying on the surface. Therefore, if the segment under consideration belongs to layer $\mathrm{j}=1$ the segments in this layer will necessarily belong to molecules of the species $N_{1}, N_{2}, \ldots, N_{v-1}$; if it belongs to layer $\mathrm{j}=2$, in this layer will be segments belonging to molecules of the species $N_{1}, N_{2}, \ldots, N_{v-2}$, and so on.

\section{Calculations}

The total interaction energy of the system and Helmholtz's free energy are given by, respectively:

$$
\begin{gathered}
U_{\text {Total }}=\Delta U_{S}+\Delta U_{\text {down }}+U_{\text {Air }} \\
F_{\text {Total }}=U_{\text {Total }}-T \Delta S_{\text {Total }}
\end{gathered}
$$

The surface pressure is calculated using:

$$
p=-\left(\frac{\partial F_{\text {Total }}}{\partial A}\right)=-\left[\frac{F_{\text {Total }}\left(N_{0}\right)-F_{\text {Total }}\left(N_{0}-\Delta N_{0}\right)}{w N_{0}}\right]
$$

where $w$ is the area of one site. To determine the equilibrium distribution, i.e,. the $N_{i}$ 's that satisfy Equations 1 and 2 and minimize Equation (12), we developed an algorithm for each value of $N_{0}$. The following steps are taken:

1) The first value of $N_{0}$ must be equal to $M$, i.e., only the head groups must lay on the surface.

2) The value of $N_{0}$ must be increased by a small amount $\Delta N_{0}$ (instead a compression we perform an expansion). The key reasoning behind this procedure is that the values of the variables $N_{i}$ 's must change in a small amount for each stage of expansion. So, instead calculating all possible values of the $N_{i}$ 's that satisfy Equations (1) and (2), it is possible to establish a range of feasible values for each $N_{i}$ given the values of the former step. For this reason (less time consuming calculations), this procedure diminishes enormously the computational effort to calculate the equilibrium distribution for all possible values of the number of sites.

\section{RESULTS}

Results show that for any size, i.e, different values of $v$, of the molecules there is at most one phase transition depending strongly on the energy parameters. Fig. 2 shows a typical result where the calculation was done for 600 molecules, each one with two segments ( $v=2$; molecules having larger number of segments showed similar results). When the area of the surface corresponds to about 800 sites, the van der Waals kind loop shown in Fig. 2 indicates that a first order phase transition takes place. This conclusion is supported by the plateau present by the Helmholtz free energy of the system around this number of sites as can be seen in the same Figure.
In Fig. 3 we show the number of molecules of each species $\left(N_{w}, N_{1}\right.$ and $\left.N_{2}\right)$ at each step of the compression with energy parameters close to those employed in Fig. 2. The hydrophobic segments lift continuously with diminishing area while the water molecules are driven continuously out of the surface down into the bulk of water. When the site number is close to 2300 half of the molecules have their hydrophobic tail expelled from the interface and as the number of sites diminishes, the number of molecules having two segments, $N_{2}$, becomes smaller while those having only one segment increases. At the phase transition (number of sites, $N_{0}$, approximately 800 ) about $85 \%$ of the molecules have their hydrophobic tail lifted.

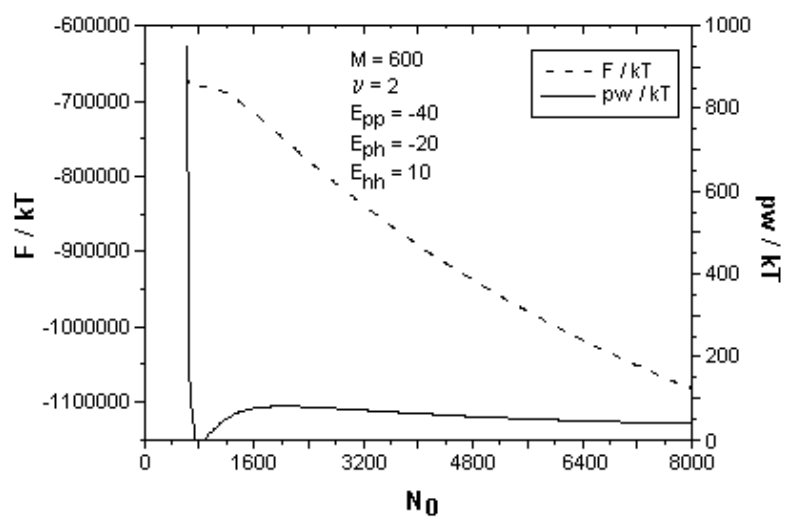

FIG. 2: Surface pressure, $p$, and Helmholtz free energy, $F$, in units of $\mathrm{kT}$, vs. number of sites, $\mathrm{N}_{0}$, for molecules of size $\mathrm{v}=2 . w$ is the size of the lattice cell. The values of the energy parameters employed are given in the Figure.

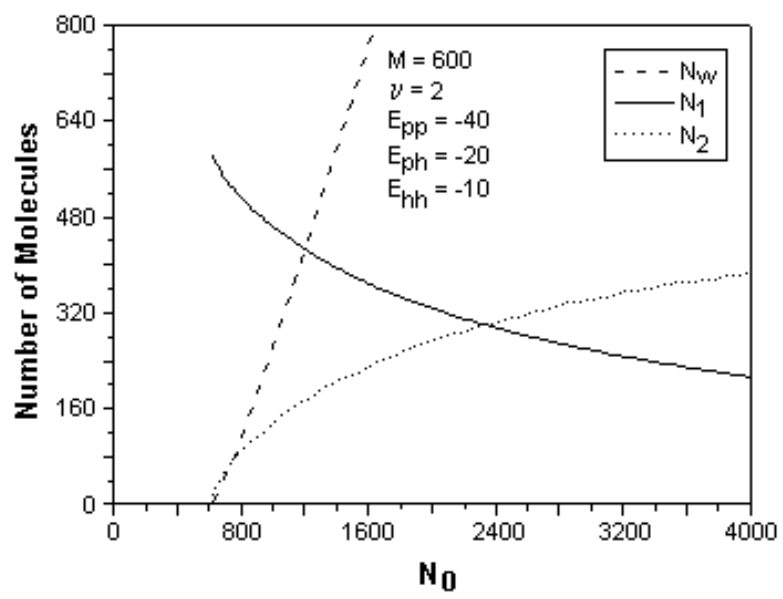

FIG. 3: Number of molecules of each different molecular species, $\mathrm{N}_{w}, \mathrm{~N}_{1}, \mathrm{~N}_{2}$, vs. number of sites, $\mathrm{N}_{0}$, for molecules of size $\mathrm{v}=2$. The parameters employed are close to those employed in Fig. 2.

As already mentioned, molecules of larger size, i.e., $v>2$, or larger number of molecules, i.e., $M>600$, show similar results under compression. This leads to the conclusion that 
the phase transition is from a phase in which the hydrophobic tails are laying on the surface to a phase where they are lifted above the surface into the air.

\section{CONCLUSIONS}

The model presented in this paper is able to reproduce only one fluid-fluid phase transition. The mechanism responsible for this transition is due to the interaction among the hydrophobic segments that at compression are gradually lifted above the surface. There are two reasons to consider concerning the absence of a second phase transition. All models that reproduced two phase transitions are hybrid models, i.e., they cannot be considered as pure lattice models, since additional hypothesis besides the lattice hypothesis, like the tilt angle of the hydrophobic tails, are used. The second reason may be the assumption of rigid rods for the hydrophobic tails driven out of the surface into the air under compression. Schmid and Schick [16] and Schmid [17] showed that the two phase transitions occur only for two sufficiently flexible chains above the surface and that the increase of rigidity gives rise to only a single transition from a gaseous to a condensed phase. Therefore one may conclude that pure lattice models are able to predict only one fluid-fluid phase transition on Langmuir monolayers.
[1] J. Harris and S. A. Rice, J. Chem. Phys. 88, 1298 (1988).

[2] J. P. Bareman and M. L. Klein, J. Phys. Chem. 94, 5202 (1990).

[3] S. Karaborni and S. Toxvaerd, J. Chem. Phys. 97, 5876 (1992).

[4] D. R. Swanson, R. J. Hardy, and C. J. Eckhardt, J. Chem. Phys. 99, 8194 (1993).

[5] F. M. Haas, R. Hilfer, and K. Binder, J. Phys. Chem. 100, 15290 (1996).

[6] Z. G. Wang and S. A. Rice, J. Chem. Phys. 88, 1290 (1988).

[7] J. M. H. M. Scheutjens and G. J. Fleer, J. Phys. Chem. 83, 1619 (1979).

[8] J. Popielawski and S. A. Rice, J. Chem. Phys. 88, 1279 (1988).

[9] S. Shin, Z. G. Wang, and S. A. Rice, J. Chem. Phys. 92, 1427
(1990).

[10] R. S. Cantor, P. M. McIlroy, J. Chem. Phys. 91, 416 (1989).

[11] P. J. Flory, J. Chem. Phys. 10, 51 (1942).

[12] A. Rusanov, J. Chem. Phys. 120, 10736 (2004).

[13] D. Volhardt, V. B. Fainerman, and F. Liu, J. Phys. Chem. B. 109, 11706 (2005).

[14] V. B. Fainerman, D. Volhardt, J. Phys. Chem. B. 103, 145 (1999).

[15] E. Ruckenstein and B. Li, J. Phys. Chem. B. 102, 981 (1998).

[16] F. Schmid and M. Shick, J. Chem. Phys. 102, 2080 (1995).

[17] F. Schmid, Phys. Rev. E 55, 5774 (1997). 